\title{
"Estado de la justicia y de la seguridad pública en Costa Rica. Aproximación comparativa con El Salvador." 1
}

\author{
Cecilia Sánchez Romero \\ Ministra de Justicia y Paz de Costa Rica
}

Mi pretensión es exponer algunas ideas generales sobre los desafíos de la justicia penal y sobre los desafíos de los sistemas penitenciarios y otros temas sobre seguridad pública, particularmente relativos a mi país, con la intención de poner en contexto y poder hacer alguna aproximación, a través de la participación que Jaime Martínez puede hacer, a la realidad de El Salvador, que, lamentablemente, hoy puedo constatar es un tema bastante sensible que plantea un enorme desafío.

Desde el punto de vista de la ciencia penal, indiscutiblemente, el problema carcelario es una derivación de una concepción específica de derecho penal, de un modelo de justicia penal y de una política criminal, si bien no articulada como tal en algunos casos; cualquier manifestación ligada a los fenómenos de la criminalidad son, sin duda, manifestaciones de la política criminal.

En Costa Rica, al igual que en muchos de los países de Latinoamérica en los últimos 10 años, prevaleció la doctrina neo-populista, lo que conocemos como el neo-punitivismo, el populismo punitivo, que optó por desarrollar políticas altamente represivas para enfrentar los temas de la criminalidad. Esto ha sido uno de los factores que ha generado que nuestro país tenga hoy día en sus centros carcelarios a una enorme cantidad de jóvenes sancionados por la comisión de delitos de muy poca monta.

Frente a cierto auge de hechos de criminalidad fundamentalmente contra la propiedad, el país abandonó las figuras penales contravencionales, que tenían unas penas sustancialmente menores a la de los delitos, aplicando la racionalidad y la proporcionalidad en el uso de la sanción penal. Esto nos permitía tener una administración de justicia de baja contención, que resolvía una gran cantidad de conflictos, entre ciudadanos, por pequeños hechos contra la propiedad, cometidos muchos por jóvenes, a quienes librábamos del ingreso a la prisión porque podíamos, atendiendo a esa proporcionalidad, aplicar medidas conciliatorias. Es decir, teníamos una respuesta dentro del orden penal; y quiero dejar esta idea planteada porque después veremos que los detractores del modelo penal garantista acusan a quienes pensamos de esta manera de que queremos generar

1 Conferencia impartida en la Academia Nacional de Seguridad Pública, ANSP, Santa Tecla, el 4 de marzo de 2016, al cuerpo docente, policial y personal profesional administrativo de la ANSP. 
impunidad, no sancionando debidamente las conductas delictivas. Esto es absolutamente falso.

De lo que se trata es de buscar racionalidad en el uso de la prisión. Porque la racionalidad tiene un impacto enorme sobre las futuras carreras delictivas de nuestros jóvenes. Efectivamente cuando el país echa marcha atrás en su política racional en la utilización del derecho penal allá por el 2004 y convierte en delitos las sustracciones de hechos de escaso valor, así como los daños, empieza a generar una cultura represiva, que opta por la pena privativa de libertad como la sanción primordial.

El incremento de esos hechos delictivos, ahora conocidos como delitos, provocó una reforma procesal allá por el 2009, que es la creación de los llamados "tribunales de flagrancia". Estos tribunales nacen en Costa Rica con una finalidad muy distinta a la que cumplen hoy día. Se establecen, precisamente, para dar una respuesta inmediata, rápida, una justicia pronta y cumplida a estos hechos en los cuales normalmente toda la prueba estaba allí; eran detenidas las personas cometiendo el hecho delictivo de manera flagrante, se detenían, pasaban a un tribunal, allí mismo se juzgaban.

Sin embargo, lamentablemente, este proceso ha degenerado y hoy día es una auténtica fábrica de condenas. ¿Por qué razón se convierte en una fábrica de condenas? En primer lugar, porque la mayoría de los hechos delictivos contra la propiedad ocurren en perjuicio de grandes empresas transnacionales; las cadenas de supermercados son las principales "víctimas" de esos hechos delictivos y las administraciones de estos negocios exigen a la administración de justicia la aplicación de sanciones; niegan la posibilidad de cualquier acuerdo conciliatorio. Esta situación se produce ahora que esos hechos son criminalizados como delito, si hubiéramos mantenido la criminalización bajo la figura de las contravenciones, se hubiese llegado a un acuerdo conciliatorio, un acuerdo reparatorio y hubiéramos impedido el ingreso de jóvenes a los centros penales, pero mediante una reforma penal de hace unos años, se convirtieron esas conductas en delitos.

La población carcelaria de Costa Rica es ubicable, en su gran mayoría, entre los 18 y los 33 años. Es decir, población sumamente joven, en edad productiva, en condiciones de estar estudiando y, sin embargo, esta es la población que está ingresando a la prisión. Entre los hechos delictivos que califican, según este nuevo concepto de justicia penal, para ingresar a prisión tenemos los absurdos de sancionar la sustracción de objetos cuyo valor, en muchos casos, no pasa de tres o cuatro dólares. El juicio para determinar esta responsabilidad penal le cuesta al país alrededor de cinco o seis mil dólares, por los costos que tienen fiscal, jueces, defensores, custodios, equipo técnico, etc. Esta realidad nos confirma, en 
buena medida, la irracionalidad del sistema penal frente a determinadas conductas.

Yo sé que en algunos países, incluida la República de El Salvador, Ios hechos criminales tienen otra monta, pero entonces tenemos que ir pensando en la conformación de una normativa penal que efectivamente establezca la pena privativa de libertad para aquellos hechos que vulneren de manera significativa la armónica convivencia. ¿Cuál es la razón de esta necesaria revisión de la política criminal?

En primer lugar, porque ninguno de nuestros Estados tiene la capacidad ni técnica ni económica para dar respuesta al ingreso tan masivo de personas privadas de libertad. Solo para tener un dato, nosotros recibimos - con nosotros me refiero al Ministerio de Justicia, que administra todo el sistema penitenciario- 620 personas al mes, entre personas indiciadas y personas sentenciadas y solo logramos egresar, a través de mecanismos sobre los cuales voy a conversarles ahora, un promedio de 200 personas.

Esto significa que siempre habrá 400 personas haciendo rezago de acuerdo con la capacidad de los centros de reclusión carcelaria. Este detalle es importante porque vamos a ver cuál es la respuesta y la reacción del sector más conservador en cuanto al pensamiento penal y, por supuesto, el sector político dominante en nuestro país, que tiene una visión absolutamente represiva, conservadora, que se niega a ligar el tema de la criminalidad con sus verdaderas causas, con sus raíces; se niega a entender que en nuestros países el tema de la criminalidad está ligado, en buena medida, a la inequidad social.

La mayoría de nuestros jóvenes en prisión no han terminado siquiera su escuela primaria, provienen de hogares desintegrados, donde no hay escuela, no hay casa, no hay trabajo, mucho menos acceso a la cultura y al deporte. Esta forma de analizar, si lográramos concientizar, para analizar el hecho, el delito, desde la óptica que lo liga a su verdadera entraña, a su raíz, implicaría que el país tendría que pasar por una revisión de su política económica y, como todos sabemos cuándo se tocan los intereses económicos de sectores poderosos, las reacciones son muy fuertes y las respuestas muy violentas y muy difíciles.

Hoy día, para compartirles nuestra realidad, estamos empeñados desde el actual gobierno en una reforma fiscal, una reforma tributaria absolutamente necesaria para evitar la evasión fiscal, para sancionar el fraude fiscal, porque ocurre en la mayoría de nuestros países, que los sectores más poderosos son los que menos tributos pagan, proporcionalmente hablando, es decir, los que más riqueza tienen pagan menos impuestos con respecto de lo que poseen. La mayoría de los tributos provienen de lo que se les retiene en planilla a los empleados privados y a los funcionarios públicos. Sin 
embargo, el costo político que ha tenido la discusión de esta reforma es que los sectores que la resisten enfilan sus cuestionamientos a la imagen del gobierno y particularmente del señor presidente de la República en este momento.

En este juego perverso de intereses económicos, que se amarran para impedir transformaciones que podrían generar condiciones más favorables para el desarrollo de los sectores muy vulnerables, también juega un papel muy importante el factor mediático. Los medios de comunicación se convierten en nuestro país -y creo que en la mayoría- en aliados de las corrientes más represivas, porque realmente esa es la noticia que vende, el hecho de sangre, la fotografía en primera plana y de allí el cuestionamiento de las autoridades por su ineficacia en la solución de los conflictos.

En Costa Rica ha habido —lamentablemente tengo que admitirlo_ - un incremento en los últimos cuatro años de la tasa de homicidios, pasamos de estar en una tasa de 8 homicidios por cada 100,000 habitantes a 11 , en este momento, que para nosotros es un aumento significativo. Ha habido un incremento, también, de hechos delictivos ligados al crimen organizado, que es una nueva modalidad en nuestro medio de participación delictiva, que también tiene que movernos a tomar otro tipo de decisiones. Es decir, tenemos que marcar una clara diferencia entre lo que significa la criminalidad de bagatela, la criminalidad de poca monta, que es la que nos tiene colapsados los sistemas penitenciarios y otro tipo de criminalidad frente a la cual el enfrentamiento tiene que mejorar las herramientas y tiene que ser distinto.

No podemos enfrentar a la criminalidad organizada con las mismas herramientas con que se enfrenta la criminalidad convencional, así como la criminalidad de cuello blanco o los grifos de corrupción que también lamentablemente se han producido los últimos años. Ustedes recuerdan que Costa Rica juzgó a dos expresidentes de la República; sentencias que fueron importantes porque fueron, de alguna manera, un buen mensaje a la colectividad, en el sentido formal al menos, de que la ley podía también llegarle al que tenía poder y recursos. Recuerden que el efecto simbólico del derecho penal, a veces, es perverso, pero, a veces, cumple un papel importante; en este caso, el caso costarricense, fue significativo el juzgamiento de estas personas. Pero, les repito, ese no es el promedio de personas que están en las prisiones costarricenses, nuestros presos siguen siendo, por lo general, pertenecientes a los sectores más carenciados.

En este contexto, para poder avanzar o para poder resolver algunos de los problemas que plantea este enfoque de derecho penal, que se traducen, en lo que al Ministerio concierne, en un alto nivel de hacinamiento, aunque sé que, comparativamente con El Salvador, no lo es tanto, pero nosotros estábamos en julio del 2015 con un $55 \%$ de hacinamiento, Io 
cual nos puso frente a la Corte Interamericana de Derechos Humanos con 14 denuncias por violación de derechos humanos, por violaciones de hacinamiento carcelario. Esta cifra, si bien es cierto, no es tan alarmante en relación con otros países, para nosotros sí lo era porque nuestro país hasta el 2004 no tenía niveles de hacinamiento superiores al $20 \%$, que es la tasa de hacinamiento crítico permitida por los organismos de derecho internacional.

Un elemento fundamental para poner en evidencia la gravedad de la situación lo es, para nosotros, el hecho de que la Convención Americana de Derechos Humanos fuera firmada en San José y que esta ciudad es la sede de la Corte Interamericana de Derechos Humanos; por tanto, nos confronta día a día frente a una realidad en la cual nosotros alegábamos ser un Estado de Derecho, un país respetuoso de los derechos humanos y el hacinamiento carcelario nos estaba reflejando como todo lo contrario.

En esas condiciones empezamos por cuestionarnos cuáles son los desafíos que tiene el sistema penitenciario para resolver los problemas que le plantea la sobrepoblación. Y creo que aquí es que la experiencia compartida vale, la sobrepoblación, el hacinamiento carcelario no puede ser el resorte exclusivo de uno de los poderes del Estado o de uno solo de los ministerios. Es decir, en la solución de los problemas carcelarios, en la respuesta a los problemas de la política criminal, tienen que participar una gran cantidad de actores. En primer lugar, tiene que haber una política pública de Estado, una política criminal, que tiene que construirse sobre la base de la participación de los tres poderes del Estado, la academia, las organizaciones no gubernamentales, los medios de prensa, de la ciudadanía y de las propias personas privadas de libertad.

Es urgente y necesario tomar el diálogo a partir de un documento base, que marque la ruta de lo que debe ser una política criminal en un Estado de Derecho. Pero entendiendo que la política criminal también se nutre de la política económica, de la política de salud pública, de la política de educación, de la política de cultura y de la política del deporte, porque el derecho penal interviene cuando todas las otras instancias del Estado han fracasado. Ustedes podrán comprobarlo en la realidad de su país, en aquellos lugares en los cuales la presencia del Estado es más escasa, es muy posible que tengamos mayores conflictos. Cuando hablamos de presencia estatal no nos referimos a la presencia policial, nos referimos a la presencia del Estado atendiendo las demandas de sus ciudadanos, asegurándoles el derecho a la educación, a la salud, a la vivienda, a la recreación y al deporte, a toda su juventud, a todos sus habitantes.

Lograr esta articulación interinstitucional no es fácil, el Poder Legislativo generalmente es resistente a la participación en los procesos de interacción con otros poderes. Recordemos que el Legislativo tiene a su favor la 
potestad de la decisión de cuáles son las conductas a las cuales atribuye una pena, en otros términos, tiene la potestad para decidir qué criminaliza y qué no. Pensamos en las realidades de nuestros países, en qué momento nuestros legisladores, a la hora de determinar que conductas delictivas sancionan, toman en cuenta la realidad económica y social del país, las posibilidades reales que tiene el sistema de justicia para dar respuesta a una excesiva criminalización, las posibilidades reales que tiene el sistema penitenciario para recibir en su seno a todas las personas privadas de libertad. Ese es un ejercicio que tiene que ser compartido, no se puede tomar decisiones de política criminal sin estimar los recursos con los que puede contar el país para hacerle frente.

Una preocupación que me surge ahora y lo digo con el mayor de los respetos, que quiero compartir con ustedes, estuve ahora en la mañana en una bartolina y quedé muy impactada por la situación tremenda de hacinamiento en ese lugar, en un espacio en el que vi que coexistían personas indiciadas y sentenciadas, lo cual es un problema que hay que resolver en algún momento; tendrá la Policía Nacional Civil que valorar si le corresponde la custodia de personas sentenciadas por un juez, tenerlas en sus instalaciones, sin poder además dotarlas de los requerimientos necesarios, la alimentación, la cama; es decir, no existe esa posibilidad, no hay recursos de la institución del Ministerio de Seguridad, porque esa no es su función obviamente, pero de hecho se cumple. Entonces, allí tenemos un tema en el cual necesitamos una articulación muy importante entre instituciones del Estado.

Cuando la Policía Nacional Civil interviene en una comunidad y desarticula una estructura criminal, se lleva presos a sus cabecillas y desaparece, ¿quién se hace cargo de las familias que quedan allí? Y lo digo porque es también nuestra realidad. Solo estamos acostumbrados a ver la eficacia policial, en muchos casos muy buena, desarticulando, pero nos falta el resto del Estado, con presencia ahí, tratando de darle seguimiento, determinando si los niños de ese hogar están en la escuela, si tienen que comer, si tienen casa. En pocas palabras, cuál es la situación que queda luego del operativo policial. Esta es una de las principales debilidades que enfrentamos nosotros en general en nuestros países, en el tema del enfrentamiento efectivo frente a hechos de criminalidad. Lo que provoca, de manera evidente, que retiramos una estructura, pero dejamos allí el semillero de quienes van a sustituirlos, pues en realidad nos dedicamos a cortar las ramas del árbol. Esto tenemos que verlo con sentido autocrítico, nos pasa a todos, porque el enfrentamiento o el enfoque de la política criminal ha carecido de esa integralidad. La política preventiva tiene que darse la mano con la política de persecución y con la política represiva.

Por otra parte, tenemos el tema de cómo las condiciones de prisionalización, nos provoca que, lejos de generar el efecto re, la falacia re, como llama el 
profesor Zaffaroni, rehabilitador, resocializador, reinserción, ninguno de todos lo cumplimos. La forma en la que estamos recluyendo a nuestra población penitenciaria es un componente más bien activo generador de violencia y esta es una de las cosas que más nos cuesta a nosotros en el país hacerla comprender, sobre todo a los señores diputados y otros políticos.

Invertir en el sistema penitenciario es invertir en seguridad, porque en la medida en que el sistema penitenciario pueda dar a su clientela, a las personas que tiene bajo su control, un abordaje técnico, integral, en la medida en que pueda dotar a los centros de reclusión de los espacios para el estudio, para el trabajo, para la recreación, puede egresar personas que más fácilmente se puedan insertar socialmente. Pero la manera en que los estamos egresando hoy, por supuesto, no tiene nada que ver con principios de inserción social. Esto nos lleva, entonces, a ver que tenemos que replantear el enfoque del trabajo penitenciario y esta revisión pasa fundamentalmente por una revisión del modelo de infraestructura carcelaria que hemos venido construyendo.

Nosotros tuvimos una construcción carcelaria en los años ochenta cuando Costa Rica aplicaba el modelo progresivo del tratamiento penitenciario, que ya no llamamos tratamiento sino atención y abordaje porque el tratamiento nos lo ligaban a un tema terapéutico, médico. Construimos espacios carcelarios que vistos hoy son verdaderas jaulas para recluir personas y el modelo de atención entonces se adecuó a la infraestructura penitenciaria. Cuando debemos de partir exactamente de lo contrario. Esto es, la infraestructura carcelaria debe responder al modelo de atención. Claro que eso nos va a costar ahora que ya hay muchas cárceles en muchos países, pero si esa infraestructura está y no responde a ese modelo, al menos tenemos que hacer el esfuerzo de orientar el abordaje del sistema penitenciario hacia esas pautas que permitan la generación de herramientas para egresar al mundo libre.

Aquí quiero hacer una observación, el trabajo que vi ayer en el modelo de Apanteos "Yo cambio" me pareció una experiencia extraordinaria en la medida que, pese a que hay una infraestructura que no se presta para este abordaje, hay un proceso y una actitud de compromiso de funcionarios que sí permite desarrollar actividades. Me impresionó mucho ver en un gran espacio abierto, sin comodidades para ser convertidos en aulas o talleres, grupos importantes de personas privadas de libertad recibiendo su capacitación, su formación, con lo cual se nos demuestra también que hay que ir acomodándonos a las circunstancias, en general, ser creativos, partir de las condiciones reales y producir modelos distintos de atención. Creo que esto es una experiencia sumamente importante que ojalá pudiera replicarse en muchos otros centros. 
Un elemento adicional al problema penitenciario son los pocos espacios que hay para el abordaje técnico, para aulas, para talleres, para el deporte, espacios para recibir la visita, techados en lugares donde llueve mucho, o en lugares donde hace mucho sol, cuántas de nuestras personas privadas de libertad en algunos lugares deben de recibir la visita en su propio dormitorio, porque no disponen de esos espacios.

Luego les voy a compartir mi experiencia con las unidades productivas que estamos ahora implementando con un empréstito del BID, que apunta a un concepto distinto de infraestructura, para posibilitar ese egreso que impida que adicionalmente, las personas privadas de libertad cuando salen -me imagino que a ustedes les ocurre lo mismo que a nosotrostienen su hoja delincuencial manchada. Esto quiere decir, está certificado el juzgamiento y prescribe después diez años. De manera que hay un doble castigo: la persona cumple su condena en el centro penal, egresa, pero tiene un castigo adicional y es que su hoja de antecedentes penales esta manchada y ¿qué empresa va a estar interesada en contratar personas que vienen egresando de la prisión aunque hayan cumplido su sentencia?.

Aquí tenemos una tarea sumamente importante de articulación con el Poder Legislativo y les comparto la experiencia nuestra: acabamos de presentar ante las sesiones extraordinarias de la Asamblea Legislativa un proyecto de ley que les podemos compartir con mucho gusto, que gradúa el registro judicial de antecedentes. De manera que no son diez años para todos los delitos, sino que vamos graduándolo de acuerdo con la naturaleza de los hechos delictivos y además elimina el certificado de antecedentes para efectos laborales. Claro que mantenemos algunas restricciones, por ejemplo, las personas de crimen organizado, de tráfico internacional de drogas, de trata de personas o de delitos sexuales, violentos, sí están excluidos. Esta es una herramienta que esperamos que posibilite que el trabajo que se pueda hacer dentro de los sistemas penales no se vea luego obstaculizado por la incorporación en el mercado laboral.

Otro proyecto de ley - con lo cual estoy comentando las alternativas que estamos valorando en este momento- que se acaba de presentar es el proyecto de ley que recupera algunas contravenciones, vuelve a establecer la proporcionalidad en el uso de la sanción y además establece un catálogo de penas alternativas. En este tema sí creo que hay que hacer un gran trabajo, desde la academia con los estudiantes y con la sociedad. Demostrar que existe una variedad de alternativas a la pena de prisión que también pueden permitir encontrar reparación del daño causado sin causar los efectos tan dañinos y tan irreversibles, que genera la pena de prisión. Sobre esto hay que trabajar con la sociedad, con los medios de comunicación, también con las universidades, con las escuelas y con los colegios. En esa dirección, el Ministerio de Justicia le presentó al Ministerio de Educación Pública un proyecto para elaborar una malla curricular que 
nos permita desde la escuela primaria en la materia de Cívica, que haya un espacio para la formación en lo que significa el sistema penitenciario y el respeto a los derechos humanos. Por supuesto orientado no en la dirección de generar temor por la cárcel, sino expresar su realidad, pero también dar a conocer las otras posibilidades que el sistema penal puede tener.

¿Qué otras alternativas ha desarrollado el país para hacerle frente al sistema penitenciario? Una medida de emergencia que tomamos en el mes de septiembre del año pasado y que ha generado una reacción política tremenda —todos los días piden mi cabeza, pero ya yo estoy acostumbrada-, la Asamblea Legislativa me hizo comparecer cinco horas ante el Pleno para explicar la medida, que simplemente consistió en algo tan sencillo como cumplir las sentencias que la Sala Constitucional y los juzgados de la ejecución de la pena han dictado. Nosotros tenemos, al igual que ustedes, el control judicial de la ejecución de la pena, en ese sentido, la Sala de lo Constitucional le ha dicho al Ministerio de Justicia en más o menos veinte sentencias, o impide el ingreso de más personas en determinados centros o a usted, señora ministra, la vamos a acusar por desobediencia a la autoridad. Las sentencias de la Sala Constitucional son vinculantes, yo estoy obligada a cumplirlas, no tengo espacios carcelarios donde recluir a las personas, tengo que dar respuesta a estas demandas, tengo que responder ante la Corte Interamericana de Derechos Humanos. Ante este panorama, emitimos una circular, la 5-2015.

¿En que consistió la circular número cinco? En establecer una serie de criterios de carácter técnico que nos permitiera reubicar a la población privada de libertad del régimen cerrado institucional al régimen semi institucional. En el régimen semi institucional que ustedes también tienen, las personas pueden llegar a dormir, un día, dos días o toda la semana, pero durante el día pueden buscar trabajo o pueden estudiar, hacer otra cosa. El perfil que se determinó para esta salida era el de personas primarias con sentencias menores a 6 años. Y, vean como nuestra población está por hechos delictivos que no son tan graves, que pudimos sacar, hemos podido sacar muchísima gente que tuviera un soporte familiar, una oferta laboral y ahora ya cedimos que como sabemos que cuesta conseguir trabajo, entonces los dejamos que salgan para que consigan el trabajo, porque es tan perverso el sistema penal que los obligamos a que tengan un trabajo y cómo va a conseguir el trabajo alguien estando privado de libertad.

Una población que también nos provoca hacinamiento, es la que está detenida por el no pago de pensiones alimenticias, es una población de 350 personas que no trabajan y que, sin embargo, está allí porque no pagan la pensión alimenticia. Es uno de los mayores contrasentidos, pero sobre ellos yo no puedo tomar ninguna decisión porque están a la orden de los juzgados de pensiones alimentarias. 
La aplicación de la circular nos permitió bajar el año pasado de septiembre a diciembre el hacinamiento a $44 \%$, es decir, egresamos 940 personas, con un costo político personal muy alto, que yo no tengo ningún inconveniente en asumir porque yo no soy política. En realidad yo estoy allí por un accidente del destino, no tengo ninguna aspiración política posterior, de manera que seguimos planeando que esa sea la respuesta.

¿Por qué se opone la ciudadanía costarricense? Porque, en primer lugar, hay una distorsión total y absoluta de lo que significa la persona privada de libertad, se consideran ciudadanos no de primera, ni segunda, ni tercera sino de quinta categoría, no merecedores de ninguna oportunidad. Nosotros, así como sabemos que hay personas de conducta altamente violenta y que no podrían estar en libertad, porque la convivencia les resulta muy difícil, hay una gran cantidad de personas, hombres y mujeres, que sí podrían estar afuera cumpliendo otro tipo de medida.

En el caso de las mujeres privadas de libertad, en Costa Rica la mayoría lo están por venta de droga al menudeo o ingreso de droga al centro penitenciario. La venta de droga al menudeo ocurre en hogares donde la madre es cabeza de familia, donde tiene varios hijos, no tiene escolaridad, no tiene ninguna profesión y ella tiene que hacerle frente a la obligación de su familia. Con estas mujeres estamos aplicando unos criterios bastantes flexibles de reubicación porque consideramos que en el contexto no constituyen mayor peligro y estamos haciendo mucho más daño a la familia teniendo a las mujeres retenidas que si ellas estuvieran al frente de sus hogares.

Una alternativa pendiente también como propuesta es el uso de los dispositivos electrónicos, los famosos brazaletes electrónicos. Si ustedes no han entrado a esa discusión, es importante a la hora de considerarlo ver las experiencias de otros países. Nosotros no hemos podido implementar el sistema pese que ya la ley tiene dos años. En primer lugar, porque la ley estableció criterios muy preceptivos para la aplicación, lo que provoca que el universo de posibles candidatos es reducido y luego la ley previó un plazo de un año para hacer un plan piloto fundamentalmente con personas indiciadas.

La tecnología de los brazaletes electrónicos es bastante cara, es una tecnología que se desactualiza, como toda la tecnología de informática y requiere de una gran cantidad de personal para el monitoreo. La tecnología no existe en nuestros países, no hay empresas que se dediquen a esa materia, lo cual genera costos muy elevados con empresas extranjeras. Pero quiero alentarlos en el sentido de que puede ser una respuesta interesante, sobre todo para casos de violencia intrafamiliar, pero no es una alternativa de respuesta inmediata. Ustedes sabrán que personas condenadas por hechos delictivos muy graves, no las vamos a sacar con 
un dispositivo electrónico, entonces habría que ver qué tan grande sería el universo de posibles candidatos. En el caso de violencia intrafamiliar necesitamos la colocación de dispositivo para la mujer o para el agredido y el agresor para determinar que no haya acercamiento. La articulación policial en este caso o la determinación de la respuesta policial tienen que ser muy eficientes: tenemos que estar seguros de que donde se está monitoreando, donde hay personas con dispositivos electrónicos y se pueda mover muy fácilmente para ubicarlos.

Otro detalle, no es el Ministerio de Justicia el que decide a quien le coloca un brazalete, son los jueces de la República y nuestros jueces no siempre tienen una gran visión, son garantistas de los procesos pero a muchos les gusta la cárcel como medida de represión, la cárcel todavía está muy dentro de la cultura judicial. Entonces las alternativas para reducir los niveles de hacinamiento cada vez se nos ponen un poco más duras.

Hay un proyecto de construcción a través del empréstito del BID, del Banco Interamericano de Desarrollo; se le prestó al Gobierno costarricense 152 millones de dólares en el año 2012 durante el gobierno anterior, ese empréstito está orientado a la creación de espacios carcelarios, delegaciones policiales, siete centros cívicos por la paz, porque el Ministerio de Justicia y Paz, tiene uno de sus viceministerios que está dedicado a programas que tengan que ver con trabajos en las comunidades. Es un trabajo interesante, articulado con la Policía, donde se identifica los principales sitios violentos, lo que Ilaman mapas de calor de la Policía, donde están las principales zonas conflictivas para establecer allí, los llamados centros cívicos, que son instalaciones muy cómodas, muy modernas, donde hay espacios para el arte, la recreación, la cultura, talleres, aulas, y cada uno -pero aquí es donde tenemos que articular muy bien estos procesos- porque cada uno de estos centros requiere el concurso del resto de los ministerios. Requerimos un delegado del Ministerio de Educación para que se haga cargo de la preparación de los cursos, un delegado de Cultura para los talleres, un delegado del Instituto de Aprendizaje igual para la formación técnica, necesitamos un delegado del Ministerio de Salud porque hay campañas en temas de salud. Si no hay articulación interinstitucional es sumamente difícil poner a funcionar un centro, sobre todo en la prevención de la violencia porque entonces distorsionamos funciones, si la Policía tiene una función prioritariamente represiva es muy difícil que pueda gerenciar sola las instalaciones que son para la prevención de la violencia.

Con estos recursos del BID también se está financiando lo que denominamos unidades productivas. La unidad productiva es un nuevo concepto de estructura de reclusión, el BID dice no financia cárceles, nos financia estas unidades productivas, que son instalaciones dedicadas a la formación y el trabajo y con esto también les quiero hacer un llamado a la consideración de la conveniencia de que los centros penitenciarios no se estructuren en 
unidades que superen los 600 privados de libertad, no es que solo cárceles de 600 pero si tenemos cárceles para 4000 que al menos las podamos dividir en espacios de 500 personas; es inmanejable un centro carcelario de más de mil personas, es absolutamente inmanejable. Nosotros debemos invertir en reformar un complejo penitenciario de cuatro direcciones en las cuales tenemos 2800 personas y ahorita la vamos a tener que dividir, porque desde el punto de vista de seguridad, desde el punto de vista de atención técnica, es sumamente complejo.

Las unidades productivas son diseños productivos hechos por especialistas en diseños constructivos carcelarios, una empresa norteamericana la hizo, que privilegia los espacios de talleres, de aulas y los espacios de recreación, los espacios de dormitorios. Son espacios muy pequeños porque la pretensión es algo así como lo que está ocurriendo con ustedes en el programa Yo Cambio; la pretensión es que en estos centros solo haya personas que estén estudiando o trabajando durante el día. La selección del personal -los denominamos centros de baja contención- para permitirnos seleccionar privados de libertad con perfiles que no pongan en riesgo la seguridad ni de los capacitadores, ni de la comunidad en la cual van a estar ubicados.

Una de las dificultades más serias que ha tenido el Misterio de Justicia en este proceso ha sido la oposición de las comunidades a la construcción de centros. Las sociedades nuestras son muy hipócritas, nos piden resolver el problema del hacinamiento, nos piden que no violentemos derechos humanos y cuando se va a hacer una cárcel nadie la quiere. Es como si fuéramos a poner, y perdón pero así nos lo han dicho en algunas comunidades, un botadero de basura. Con el reflejo, el estigma, el prejuicio con lo que la población privada de libertad puede significar para algunas personas. Esto nos hizo renunciar, en el caso nuestro, a la construcción y a uno de los mejores terrenos del Ministerio de Justicia en la provincia de Guanacaste, dado que se hizo tema de campaña política, el alcalde del lugar estaba por reelegirse y su lema de campaña fue "no a la cárcel".

Nosotros podemos construir una prisión sin necesidad de autorización de la municipalidad, es un tema de interés estatal, está por encima del interés local, pero realmente no quisimos hacer un conflicto en la comunidad, porque nos amenazaban con bloquear las carreteras, con agredir a los trabajadores que llegaran a construir y dado que teníamos otros terrenos preferimos empezar a construir en otros lugares. Estos modelos de reclusión, sin embargo, son sumamente caros, a la ciudadanía y a la gente en general se le olvida que la construcción de centros carcelarios es carísima, 20 millones de dólares nos cuesta cada una de estas unidades para albergar apenas a 540 personas y 20 millones de dólares solo la infraestructura y su equipamiento. Y aquí hay que meter alrededor de 200 personas entre seguridad, personal técnico, personal de cocina, 
instructores, etc., ¿de dónde sale esa planilla? Con la situación fiscal, con la situación de ingresos que tienen nuestros países, se le olvida a la gente que esas decisiones de estar construyendo no son soluciones, no siempre son las soluciones adecuadas a nuestra realidad.

Y de nuevo volvemos al tema porque me imagino que ustedes deben de tener un nivel de ingreso parecido al de nosotros o superior. Entonces, siempre el sistema penitenciario va a ir rezagado en su capacidad para tener a la gente; luego, como decía el profesor Zaffaroni, por más cárceles que construyamos, si seguimos construyendo al final no habrá quien cierre la puerta verdad, porque todos vamos a estar en la prisión.

La lógica perversa de la prisión es la que tiene que hacernos a nosotros reflexionar sobre la impostergable necesidad de los mecanismos alternativos. El tema aquí -y con esto quiero cerrar la idea- va en tres direcciones. En primer lugar, los problemas del hacinamiento no se pueden resolver solo desde la óptica de un ministerio y de los responsables de los centros penales. Requieren un abordaje integral, requieren un abordaje de política criminal, una redefinición de las conductas a las cuales se liga una sanción, una redefinición del catálogo sancionatorio, pero prioritariamente una redefinición de las políticas preventivas que impidan que los jóvenes sigan alimentando las prisiones.

Ahorita por la mañana estuvimos en el centro penal de Izalco. Me impresionó mucho la forma en la que tienen a los niños, hijos e hijas de las personas privadas de libertad, con una atención extraordinaria, con un compromiso de las compañeras allí presentes y creo que ese es un trabajo extraordinario; en la medida en que el buen trabajo que se haga con estos niños y niñas va a impedir que traspasen los muros en los que están sus padres y sus madres. Creo que a veces se nos olvida que es allí en la parte preventiva.

Nosotros, al final, solo queremos actuar en la fase de comisión del hecho delictivo, en la fase de aplicación de la ley penal. El no dimensionar el problema penitenciario desde la óptica de un problema de política pública de Estado, que se vea atravesado por el resto de los actores, es parte de los sucesos que nos tienen entrampados con enorme crecimiento de los niveles de hacinamiento y con posibilidades enormes de habernos enfrentado a condenas en la Corte Interamericana de Derechos Humanos. Para Costa Rica sería tremendo, nos condenaron por la inexistencia de un mecanismo regulatorio de la fecundación in vitro, ustedes pudieron ver cómo la Asamblea no quería dictar la ley, el presidente elaboró un decreto, la Sala de lo Constitucional declaró inconstitucional el decreto pero la Corte Interamericana de Derechos Humanos, dichosamente, dijo que con el decreto cumplía el Poder Ejecutivo ante la inoperancia de la Asamblea Legislativa. 
Lamentablemente tengo que admitir que en Costa Rica no solo los niveles de hacinamiento en términos de sentenciados, sino también la tasa de personas privadas de libertad por cada 100000 habitantes nos acerca peligrosamente a tasas de países altísimos en niveles de encierro. Estados Unidos creo que anda llegando por alrededor de 1000 por cada 100000 habitantes; nosotros estamos ahorita alrededor de 351 personas privadas de libertad por cada 100000 habitantes, que es una tasa para nosotros exorbitante. La tasa de prisión preventiva nos ha aumentado bastante y andamos alrededor de un $24 \%$ de personas privadas de libertad por prisión preventiva.

La única "ventaja" entre comillas que podríamos encontrarle a la reclusión es que nosotros sí tenemos obligatoriamente que tener separadas a las personas sentenciadas de las personas que están en prisión preventiva. Sin embargo, también esto nos ha provocado un enorme problema de hacinamiento, solo hay una prisión para los indiciados que está, como el resto de las cárceles, abarrotada.

En fin, los retos del sistema penal, de la justicia penal y del sistema penitenciario son enormes. Las carencias presupuestarias son de los elementos que más nos afectan, carencia de recursos, incomprensión social del fenómeno y, por supuesto, un tema ligado a la estructura socioeconómica de nuestros países, que no se ha caracterizado -y me voy a referir a mi país exclusivamente- por una adecuada distribución de la riqueza. Costa Rica creció en su nivel de riqueza, pero igualmente amplió la brecha social y creo que esto nos está reflejando ese incremento lamentable que tenemos. Yo se que comparativamente con algunos países del Triángulo Norte podríamos pensar que las cifras no son tan alarmantes, pero si lo son para un país que tradicionalmente no tuvo esta clase de dificultades. Las decisiones de política de desinstitucionalización habrá que tomarlas en su momento, porque las cárceles deben de tener un límite como todo sistema, todo sistema debe tener un límite, por qué el penitenciario tiene que sobrepasarlo.

$Y$ finalmente, un temita que no quiero que se me escape, a nivel internacional incluso tenemos que analizar, que la población privada de libertad tampoco forma parte de las mayores preocupaciones de los organismos del derecho internacional. Recordemos que hay muchas convenciones de derechos humanos, de protección de las mujeres, de los niños, de las personas con discapacidad, como instrumentos obligatorios del derecho internacional. Mientras que, para las personas privadas de libertad, tenemos instrumentos de recomendación que nos marcan directrices, como las reglas mínimas, como tratamiento de las personas privadas de libertad recientemente modificadas en diciembre de este año, las reglas Nelson Mandela que, si bien implican alguna mejora en la tutela de los derechos humanos de las personas privadas de libertad, no las elevan 
a rango de instrumento obligatorio. Se deben convertir, sin embargo, en norte para nosotros, en una herramienta que nos marca un camino, nos obliga a atender las necesidades de las personas privadas de libertad, o nos obliga, por ejemplo, a tener una biblioteca en todos los centros penales, no solamente a tenerla, a firmar que se utilice por parte de las personas privadas de libertad y mejorar los principios que antes teníamos.

Pero estas reglas vienen de 1926, en 1955 se adoptan y no es hasta el 2015 que se vuelven a revisar y de nuevo siguen manteniendo el carácter de instrumentos no obligatorios, con lo cual cierro para decirles que lamentablemente la población privada de libertad, la población con la que a nosotros nos toca trabajar, a don Rodil por acá en El Salvador y a muchos de ustedes, es la población más abandonada del sistema social. Y en favor de ellos cualquier medida que se tome será altamente censurada, así es que tenemos un enorme reto si queremos dar alguna respuesta. 